\title{
Presence and strength of binding of IgM, IgG and IgA antibodies against SARS-CoV-2 during CoViD-19 infection
}

\author{
Richard B.M. Schasfoort ${ }^{\mathrm{a}, *}$, Jos van Weperen ${ }^{\mathrm{b}}$, Margot van Amsterdam ${ }^{\mathrm{a}}$, Judicaël Parisot ${ }^{\mathrm{c}}$, \\ Jan Hendriks ${ }^{\mathrm{d}}$, Michelle Koerselman ${ }^{\mathrm{d}}$, Marcel Karperien ${ }^{\mathrm{d}}$, Anouk Mentink ${ }^{\mathrm{a}}$, Martin Bennink ${ }^{\mathrm{e}}$, \\ Hans Krabbe ${ }^{\text {f,g }}$, Leon WMM. Terstappen ${ }^{\mathrm{a}}$, A.H. Leontine Mulder ${ }^{\mathrm{g}, \mathrm{h}}$ \\ ${ }^{a}$ Department of Medical Cell BioPhysics, Faculty of Science and Technology, University of Twente, PO Box 217, 7500, AE, Enschede, the Netherlands \\ ${ }^{\mathrm{b}}$ Vysens BV, PO Box 50035, 7550, LA, Hengelo, the Netherlands \\ c Carterra, 825 N. 300 W., Suite C309, Salt Lake City, UT, 84103, USA \\ ${ }^{\mathrm{d}}$ Department of Developmental BioEngineering, Faculty of Science and Technology, University of Twente, PO Box 217, 7500, AE, Enschede, the Netherlands \\ ${ }^{\mathrm{e}}$ NanoBio Research Group, Saxion University of Applied Sciences, PO Box 70000, 7500, KB, Enschede, the Netherlands \\ ${ }^{\mathrm{f}}$ Department of Clinical Chemistry and Laboratory Medicine, Medisch Spectrum Twente, PO Box 50000, 7500, KA, Enschede, the Netherlands \\ ${ }^{g}$ Department of Clinical Chemistry, Medlon, BV, 7512KZ, Enschede, the Netherlands \\ ${ }^{\mathrm{h}}$ Department of Clinical Chemistry and Laboratory Medicine, Ziekenhuis Groep Twente, PO BOX 7600, 7600, SZ, Almelo, the Netherlands
}

\section{A R T I C L E I N F O}

\section{Keywords:}

Surface plasmon resonance imaging

SPR

Affinity

off-rate

Biosensor

\begin{abstract}
A B S T R A C T
Surface Plasmon Resonance imaging (SPRi) was used to determine the presence and strength of binding of IgG, IgM and IgA against the Receptor Binding Domain (RBD) of SARS-CoV-2 in sera of 119 CoViD-19 patients. The SPRi assay measures the antibody isotype levels and the strength of binding to the RBD of ultimate 384 patient samples in one run. It turns out that during the course of the disease, the IgG levels and strength of binding increased while generally the IgM and IgA levels go down. Recovered patients all show high strength of binding of the IgG type to the RBD protein. The anti-RBD immunoglobulins SPRi assay provides additional insights in the immune status of patients recovering from CoViD-19 and this new method can furthermore be applied for the assessment of the quality of the immune reaction of healthy individuals to SARS-CoV-2 in vaccination programs.
\end{abstract}

\section{Introduction}

Corona viruses pose serious health threats to humans and animals. SARS-CoV-2 crossed the species barrier again and causes serious respiratory infections in humans. In contrast to SARS-CoV that appeared in 2003 and the Middle East respiratory syndrome (MERS) in 2012, SARSCoV-2 shows an unprecedented pandemic spread. Diagnostic tests of individuals, who are suspected of SARS-CoV-2 infection, mainly rely on real-time reverse transcription polymerase chain reaction (rRT-PCR) of viral genetic material collected in nasopharyngeal swabs [Chen C. et al., 2020, Vashist K.S. 2020]. In contrast to the PCR test, serologic assays demonstrate the presence of an immune reaction against the virus through detection of immunoglobulins directed against SARS-CoV-2 structural proteins [To et al., 2020; Montesinos et al., 2020]. The immune system of a CoViD-19 patient produces antibodies to SARS-CoV-2 within days to a few weeks following viral infection [Long et al., 2020, ]. Antibodies will remain for months following infection, as previously shown following the 2003 outbreak of SARS-CoV-1 [Cao et al., 2007] although the levels decline for SARS-CoV-2 [Beaudoin-Bussières et al., 2020]. The immune reaction to corona viruses generally provides immunity via neutralizing antibodies [Yuchun et al., 2004] in the event of a second exposure to the virus and this also provides the basis for vaccine development.

Serological antibody testing [Jin et al., 2020] is essential to get an indication whether or not an individual has been infected with SARS-CoV-2 in the past. The quality of the immune response is not only determined by the amount of antibodies but also by the overall strength of binding of the pool of potential neutralizing antibodies that binds to the relevant immunogenic proteins of the Corona virus. Recently, it was shown that the most potent, highest affinity neutralizing antibodies were directed to the receptor-binding domain (RBD) [Brouwer et al., 2020]. This spike protein RBD of SARS-CoV-2 domain binds to the angiotensin converting enzyme2 (ACE2) receptor expressed by many cell types from epithelial cells, to blood vessels, kidneys and

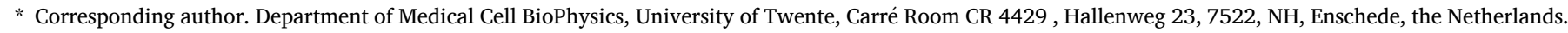

E-mail address: r.b.m.schasfoort@utwente.nl (R.B.M. Schasfoort).
} 
gastrointestinal tract, and abundant on pneumocytes-hence the respiratory symptoms. The high ACE2 binding affinity of the RBD, furin preactivation of the spike, and hidden RBD in the spike potentially allow SARS-CoV-2 to maintain efficient cell entry while evading immune surveillance [Shang et al., 2020]. The affinity of the RBD of SARS-CoV and SARS-CoV-2 to the ACE2 receptor appeared to be approximately $31 \mathrm{nM}$ and $\mathrm{K}_{\mathrm{D} \sim 5} \mathrm{nM}$ respectively as determined by surface plasmon resonance (SPR) [Lan et al., 2020]. Hence, the antibodies need to have a 6-times higher binding affinity to disrupt ACE2 mediated entry compared to SARS-CoV [Wrapp et al., 2020]. calculated affinity differences between SARS-CoV and SARS-CoV-2 to be even 10-20 times. Polyclonal supporting neutralizing antibodies preferably of high affinity $(<\mathrm{nM})$ should be raised additionally to block the RBD of SARS-CoV-2 from binding to the ACE2 receptor [Khatri et al.]. Early stages of vaccine development and clinical trials will require assessing antibody titers in combination with detecting the apparent polyclonal affinity against RBD for SARS-CoV-2.

Antibody detection is typically performed using ELISA or related automated immuno-assays. While ELISA has high-throughput capability when automated, it requires several hour-long steps that lengthen assay time. Testing of IgG, IgM and IgA requires individual assays in series for both ELISA and other immunoassays. An attractive alternative is surface plasmon resonance imaging (SPRi). SPRi is a label-free sensing technique that is highly sensitive enabling the quantitative and qualitative interaction between biomolecules in high throughput. [Schasfoort, 2017]. Furthermore similar label-free biosensor technologies as portable SPR equipments and optical fiber based detection can be applied to develop a diagnostic COVID19 test (Khansili N. et al., 2018, Wang et al., 2019).

Here, we describe a high throughput Surface Plasmon Resonance imaging (SPRi) assay for the quantitative measurement of IgG, IgM and IgA antibodies binding to the RBD spike protein and their apparent polyclonal affinity in sera of CoViD-19 patients.

\section{Materials and methods}

\subsection{CoViD-19 patient samples}

Residual serum samples ( $n=70$ ) were obtained from 53 unique CoViD-19 patients confirmed by RT-qPCR and CT-scans and collected from March 2020. In total 20 out of 70 serum samples from cases were collected within 10 days after first symptoms (range 4-9 days), 50 were collected 10 or more days after first symptoms (range 10-28 days). From 10 patients more than 1 serum sample obtained at different time points was included.

Control non-SARS-CoV-2 samples $(n=49)$ were obtained from anonymous stored residual serum samples from healthy pregnant women collected in March $2019(\mathrm{n}=37)$ and from 12 hospitalized patients with repetitive negative RT-PCR and a non-COVID explanation for their clinical symptoms. Patient characteristics are provided in MethodX supplementary Table 1.

Details regarding the immuno assays ELISA and Ig electrochemiluninescence (ECLIA) are described in the MethodX supplementary paper. Additionally sensor preparation, spotting the sera, measurements on the IBIS MX96 (Enschede, The Netherlands) and the LSA (Carterra, SLC, Utah, US) are described also in this supporting MethodX manuscript.

\section{Results and discussion}

3.1. Simultaneous measurement of 96 samples for anti-SARS-CoV-2 IgM, IgG and IgA antibodies by SPRi

Fig. 1 shows the principle of the SPRi assay for determining the isotype fractions in one run. In Panel A, 96 sera are spotted on an RBD coupled sensor. In Panel B, the sensor is placed in the SPRimager and

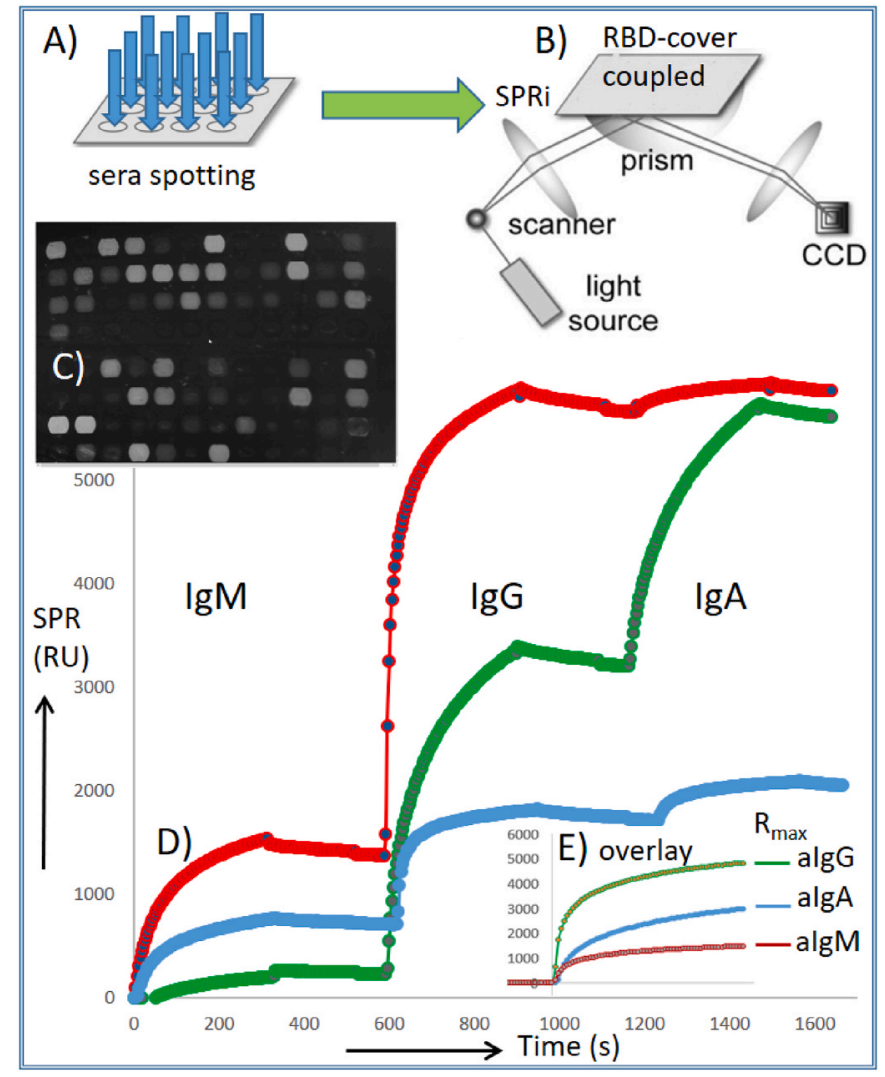

Fig. 1. Principle of the anti-SARS-CoV-2 immune globulins SPRi assay. The process of spotting the sera (A) to an RBD coupled surface in the MX96 SPRi instrument (B) resulted in a SPRi reflectivity image $(C)$. In $(D)$ the sensorgram is shown of injections of three anti-Ig to determine the response of IgM, IgG and IgA antibodies. In panel (E) an overlay of the injections of the anti-IgM (red), anti-IgA (blue) and anti-IgG (green) antibodies to a single spotted serum is presented for calculating the $R_{\max }$ values of the IgM, IgG and IgA binding, after zeroing, aligning the sensorgram.

real-time measurements were performed during three concatenated injections of anti-IgM, anti-IgG and anti-IgA. Panel C shows the SPR reflection image after the injection of the anti-isotype antibodies of the 96 sera. Panel D shows typical sensorgrams of 3 sera (red, blue, green). The red curve represents a serum with high IgM $\left(R_{\max }, 1940 \mathrm{RU}\right)$, very high IgG (5012 RU) and weak IgA (243 RU). The blue curve shows a serum with moderate IgM ( $845 \mathrm{RU})$, a moderate IgG (1215 RU) and a weak IgA (464 RU). The green curve shows a serum with a weak IgM (203 RU) a high IgG (3950 RU) and a high IgA (3796 RU). Panel E shows a patient overlay for calculating the $R_{\max }$ values of anti-IgM, anti-IgG and anti-IgA which is proportional to the bound IgM, IgG and IgA anti$\mathrm{RBD}$. The baselines are zeroed and the injections are aligned. This enables the application of a biphasic binding model for calculation of $R_{\max }$ for the three isotypes. Sensorgrams were measured for all samples simultaneously and shown as an overlay plot in the supplementary data. Repetitive measurements using the same sera showed that the RU level variation was less than $5 \%$.

In Fig. 2 the IgM, IgG, IgA and total IgG SPRi RU values are shown and samples are divided into those that are CoViD-19 PCR negative and the CoViD-19 PCR positive. The latter were subdivided into those with onset of disease symptoms of less than 10 days and those with onset of disease symptoms more than 10 days. Differences between the groups were all significant ( $\mathrm{p}<0.001$ non-parametric Mann Whitney U test). Additional data is shown in the supplementary MethodX article.

\subsection{Comparison with ELISA and ECLIA}

Using ROC curves, the optimal threshold and AUC were calculated 


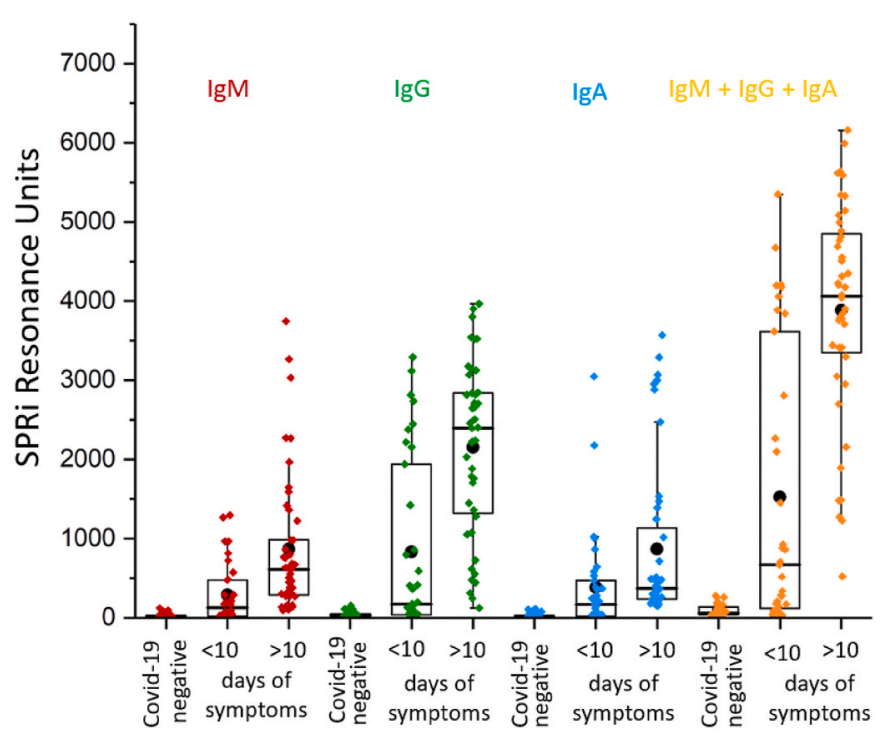

Fig. 2. The IgM, IgG, IgA and total Ig values of 36 CoViD-19 PCR negative sera, 32 CoViD-19 PCR positive sera with onset of disease symptoms of less than 10 days and 45 CoViD-19 PCR positive sera with onset of disease symptoms of more than 10 days. The box plot represents the median, p25 and p75 values and the black circle the median SPRi RU value.

for each parameter (IgG,IgA,IgM, total Ig (all SPR), ELISA IgG and ECLIA $\mathrm{Ig}$ ). The calculations were performed on 42 positive samples from positive patients and 46 samples from controls. The obtained thresholds and AUCs are shown in the MethodsX supplementary data. The calculated threshold for the ELISA was in line with the recommendations from the manufacturer, the calculated threshold for the ECLIA was lower than the recommendation from the manufacturer. With the thresholds, the sensitivity and specificity could be calculated.

The IgG SPR results were correlated with the S1 domain ELISA results and the total Ig SPR results were correlated with the ECLIA total Ig results. The correlation between IgG SPR and ELISA SPR was superior (n $=101$, Pearson's $r$ 0.95) compared to the total Ig SPR and less with ECLIA Ig $(n=116$, Pearson's $r$ 0.73). This can be explained by the fact that the ECLIA contained the nucleocapsid protein as antigen, whereas the ELISA used the S1 domain protein,. The SPRi results for anti-RBD antibodies only were correlated with clinical and laboratory parameters known to have a relation with disease activity and/or severity as CRP, ferritin, procalcitonin, lymphocyte count, lactate dehydrogenase (LD) and D-dimer. Only a slight correlation was found between the IgG SPR results and the D-dimer level. ( $n=22$, Pearson's $r=0.77)$. Although the mean level of IgG SPR was higher in patients with pulmonary embolism compared to patients without, this did not reach significance (p $=0.17$ ).

\subsection{Strength of binding measurement of anti-SARS-CoV-2 spike RBD IgG, IgA and IgM}

The on-rate for calculating the apparent affinity constant should contain the apparent concentration of the polyclonal antibodies and isotypes. We need this concentration for determining the equilibrium dissociation constant $\left(\mathrm{K}_{\mathrm{D}}\right)$ but it is impossible to determine the concentration of each clone including isotypes that contribute to the total response. However, an apparent concentration of the polyclonal mixture can be calculated from the initial slopes of the binding curves and this concentration was applied for calculating the on-rate in a kinetic serial diluted tests of injections of patient sera. (see supplementary data). We found that the affinity equilibrium constant $\left(\mathrm{K}_{\mathrm{D}}\right)$ correlated well with the off-rate $\left(\mathrm{k}_{\mathrm{d}}\right)$ and so the off-rate parameter within a certain margin is sufficient for ranking the strength of binding of the patient sera to the
SARS-CoV-2 antigens (see supplementary data). With this observation, it is now possible to rank the strength of binding or apparent affinity in high-throughput for 384 patient sera simultaneously by measuring the off-rate only. This simplifies the high throughput test enormously because the value of the apparent concentration of the anti-RBD antibodies in the patient sera is not needed for the calculation of ranking the strength of binding by the off-rate $\left(\mathrm{k}_{\mathrm{d}}\right)$.

Ligand density can cause analyte to rebind during its dissociation. The rebinding effect results in an underestimation of the off-rate value for spots with higher ligand density with respect to spots with lower density. [Schasfoort et al., 2016]. To reduce the rebinding effect of dissociating molecules, we added free RBD in a concentration of 15 $\mu \mathrm{g} / \mathrm{ml}$ to the running buffer. In 5 minutes, we observed a mixed degree of dissociation of the various and longitudinal samples (see supplementary Figure 2) and the dissociation or off-rate constant can be calculated and plotted as a function of the days of symptoms onset (see Fig. 3). During the development of the disease, we observed a smaller off-rate indicating that the avidity or quality of the antibodies improved. So, the patients are producing a better-quality repertoire of polyclonal anti-RBD antibodies over time. For all longitudinal samples this trend in off-rate is observed (strength of binding becomes better). The method is reliable, independent of concentration, high throughput and accurate for profiling the immunity of patients. Our method revealed the trends of maturation of the overall quality of the antibodies.

It is worthy of mention that this approach can be readily applied to monitoring immune response in other types of disease as well. Any protein targeted by an immune response can be immobilized to the sensor surface allowing for a very high-throughput, quantitative, and reproducible means of characterizing immunity. The real-time monitoring of signals in SPRi makes it well suited too for rapid deployment and optimization. This feature is valuable for instances where screening must be done against evolving forms of antigen, such as in the case of mutations.

\section{Conclusions}

We demonstrate a high-throughput SPR imaging platform for 384 sera suited for the rapid detection of the strength of binding of SARSCoV-2-associated antibodies of isotypes IgG, IgM and IgA. Our Surface Plasmon Resonance imaging method for profiling the immunity in terms of both isotype concentration and strength of binding to SARS-CoV-2 enables to reveal the quality of the antibodies in a high-throughput manner. High avidity anti-RBD antibodies at low concentration are perhaps more effective for neutralizing the SARS-CoV-2 and mutants of the virus [Nelson, 2021] than a higher concentration anti-RBD antibodies with lower affinity. The recent emergence in the United Kingdom and South Africa of natural variants of the virus suggests that SARS-CoV-2 has the potential to escape an effective immune response and that vaccines and antibodies able to control emerging variants should be developed. [Andreano E., 2020].

We measured 119 sera including longitudinal samples obtained from 53 unique positively PCR-tested patients with critical, severe, moderate and mild symptoms and control sera. It turns out that the off-rate (kd) parameter is sufficient to reflect the equilibrium dissociation constant (KD) of the polyclonal anti-RBD antibodies. Although the patients show a large variation in immune response composition; generally, the strength of binding showed an affinity maturation over time.

In this workflow, the maturation effect of the affinity of antibodies can be ranked and quantified precisely with the goal of improving clinical outcomes. Moreover, this assay is ideally suited for monitoring the concentration of anti-RBD antibodies of both CoViD-19 patients and healthy people who are vaccinated against SARS-CoV-2. The SPRi assay described here can provide critical insights in determining if the final quality of the IgG response after vaccination is adequate to generate neutralizing antibodies with sufficient affinity for clearing SARS-CoV-2 and its mutants to the virus. 


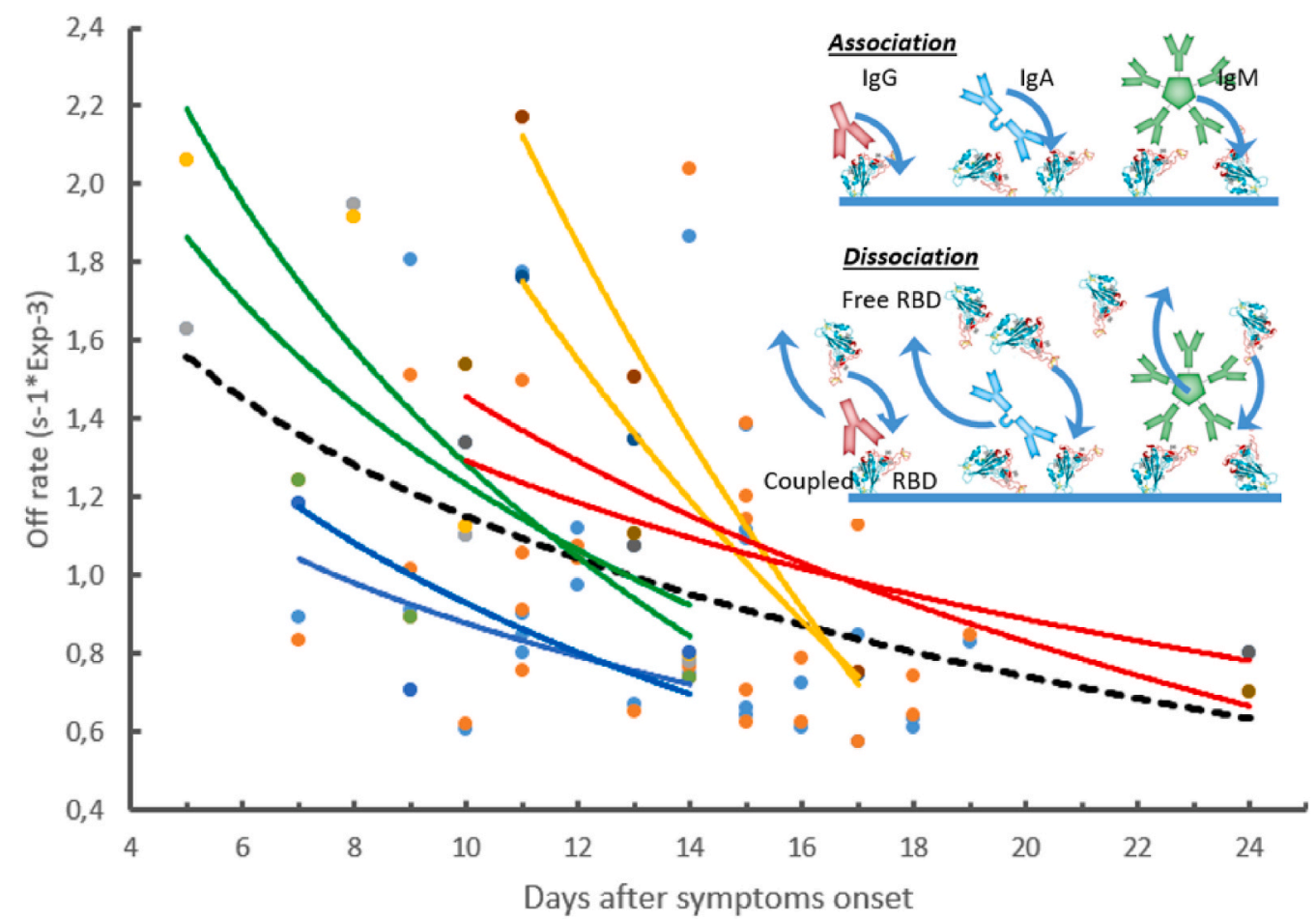

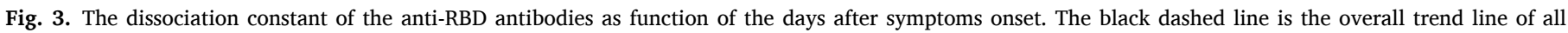
measured samples. The colored lines connect four longitudinal samples in duplicate.

\section{CRediT authorship contribution statement}

Richard B.M. Schasfoort: Conceptualization, Methodology, Investigation, Formal analysis, Data curation, Writing - original draft, Writing - review \& editing, Visualization, Project administration, Funding acquisition. Jos van Weperen: Validation. Margot van Amsterdam: Software, Data curation. Judicaël Parisot: Data curation, MK: Writing - review and editing. Jan Hendriks: Investigation, Formal analysis. Michelle Koerselman: Formal analysis. Marcel Karperien: Resources. Anouk Mentink: Formal analysis. Martin Bennink: Writing - review \& editing, Funding acquisition. Hans Krabbe: Writing - review \& editing, Conceptualization, Validation. Leon WMM. Terstappen: Resources, Writing - original draft, Writing - review \& editing, Supervision. A.H. Leontine Mulder: Conceptualization, Validation, Writing review \& editing, Supervision.

\section{Declaration of competing interest}

All (co)authors declare that they have no known competing financial interests or personal relationships that could have appeared to influence the work reported in this paper. However, except one of the co-authors Judicael Parisot of Carterra who helped us with setting up the method on the Carterra LSA SPRimaging Platform because it could not measured on the IBIS MX96 SPRimager. He set up the experiment and performed calculations for the off-rates for 384 analyses simultaneously. Because the method as shown in the paper is not (yet) embedded in the software of the equipment an enormous effort was carried out with the data to get the information we needed for the paper.

\section{Acknowledgements}

The study was performed in accordance with the guidelines for sharing of patient data of observational scientific research in emergency situations as issued by the Commission on Codes of Conduct of the Foundation Federation of Dutch Medical Scientific Societies (https:// www.federa.org/federa-english). This project has received funding from the ATTRACT project funded by the EC under Grant Agreement 777222. The Dutch Research Council (NWO), (Kirsten Ampt) is acknowledged for support of sensors and reagents.

\section{References}

Andreano, E., 2020. bioRxiv. https://doi.org/10.1101/2020.12.28.424451, 2020.12.28.424451.

Beaudoin-Bussières, G., et al., 2020. mBio 11, 5. https://doi.org/10.1128/mBio.0259020 e02590-20.

Brouwer, P.J.M., et al., 2020. Science 369, 643-650. https://doi.org/10.1126/science abc5902, 8504.

Cao aW.-C., Liu W., Zhang P.-H., Zhang F., Richardus J. H., N. Engl. J. Med., 357, 11621163. doi: 10.1056/NEJMc070348.

Chen, C., et al., 2020. Ann. Intern. Med. https://doi.org/10.7326/M20-0991.

Jin, Y., et al., 2020. Int. J. Infect. Dis. 94, 49-52. https://doi.org/10.1016/j. ijid.2020.03.065.

Khansili, N., et al., 2018. Sensor. Actuator. B Chem. 265, 35-49. https://doi.org/ 10.1016/j.snb.2018.03.004.

Khatri, I., et al., 2020. An immune perspective. Front. Immunol. 11, 570018. https://doi. org/10.3389/fimmu.2020.570018.

Lan, J., et al., 2020. Nature 581, 215-220. https://doi.org/10.1038/s41586-020-2180-5.

Long, Q., et al., 2020. Nat. Med. 26, 845-848. https://doi.org/10.1038/s41591-0200897-1.

Montesinos, I., et al., 2020. J. Clin. Virol. 128, 104413. https://doi.org/10.1016/j. jcv.2020.104413.

Nelson, G., et al., 2021. bioRxiv. https://doi.org/10.1101/2021.01.13.426558, 2021.01.13.426558.

Schasfoort, R.B.M., et al., 2016. Anal. Biochem. 500, 21-23. https://doi.org/10.1016/j. ab.2016.01.023.

Schasfoort, R.B.M., 2017. of the Handbook of Surface Plasmon Resonance, second ed. Royal Society of Chemistry London, UK.

Shang, J., 2020. Proc. Natl. Acad. Sci. Unit. States Am. 117 (21), 11727-11734. https:// doi.org/10.1073/pnas.2003138117.

To, K.K.-W., et al., 2020. The Lancet Infectious Diseases. https://doi.org/10.1016/ S1473-3099(20)30196-1.

Vashist, K.S., 2020. Diagnostics 10. https://doi.org/10.3390/diagnostics10040202.

Wang, D., et al., 2019. Sensors 19 (6), 1266. https://doi.org/10.3390/s19061266.

Wrapp, D., et al., 2020. Science 367, 1260-1263. https://doi.org/10.1126/science. abb2507.

Yuchun, N., et al., 2004. J. Infect. Dis. 190, 1119-1126. https://doi.org/10.1086/ 423286, 2004. 